\title{
Frequency of Severity of Disability in Patients with Grade III Lumbar Neural Foraminal Stenosis on Magnetic Resonance Imaging
}

Qazi Saad B. Khalid ${ }^{1}$, Raza Sayani ${ }^{2}$, Muhammad Zeeshan ${ }^{3}$, Shahbaz Alam ${ }^{3}$

1. Radiology, Dallah Hospital, Dallah, SAU 2. Radiology, Aga Khan University Hospital, Karachi, PAK 3. Radiology, Royal Blackburn Hospital / East Lancashire Hospital Trust (ELHT), Manchester, GBR

Corresponding author: Raza Sayani, sayani_raza@yahoo.com

\section{Abstract \\ Background}

Low back pain is a common condition and carries substantial socioeconomic implications. Magnetic resonance imaging (MRI) is the imaging modality of choice with lumbar neural foraminal stenosis being one of the most common causes of lower back pain syndromes. Studies have shown a lack of correlation between patients' severity of disability and radiologically determined nerve root constriction. Therefore, the goal of this study will be to determine the frequency of severity of disability in patients with severe (i.e., grade III) lumbar neural foraminal stenosis on MRI to ascertain the impact of MRI diagnosis on clinical outcomes.

\section{Materials and methods}

Two hundred fifty patients of either gender with a history of backache referred for MRI were included by purposive sampling. Of these 250 patients, 27 patients had grade II lumbar neural foraminal stenosis, and 21 had grade I neural foraminal stenosis on MRI and were excluded. Thirty-two patients had a spinal infection (e.g., tuberculosis), and 24 patients had a history of trauma. Further, 31 patients were having follow-up scans for previously diagnosed lumbar neural foraminal stenosis. Hence, after excluding these cases, 115 patients were enrolled in this cross-sectional study with grade III lumbar neural foraminal stenosis on MRI.

\section{Results}

The mean age was 51 years (range: 20 to 82 years). Most of the patients (55.6\%) were older than 50 years. The most common site of grade III lumbar neural foraminal stenosis was L4-L5 (56.5\%). According to the Oswestry disability index, 47 patients (40.9\%) had a severe disability, $32(27.8 \%)$ had a moderate disability, $16(13.9 \%)$ were diabled, 14 (12.2\%) had a mild disability, and six (5.2\%) were bedridden.

\section{Conclusions}

Received 03/02/2019 Review began 03/11/2019 Review ended 03/27/2019 Published 04/04/2019

\section{() Copyright 2019}

Khalid et al. This is an open access article distributed under the terms of the Creative Commons Attribution License CC-BY 3.0., which permits unrestricted use, distribution, and reproduction in any medium, provided the original author and source are credited.
While MRI is the imaging modality of choice in degenerative lumbar spinal stenosis, clinical disabilities can be more extensive than what radiological findings may indicate in approximately $40 \%$ of the cases.

Therefore, lumbar spinal stenosis should be a neuro-radiological diagnosis, and surgical decisions should be based on clinical scenarios in addition to MRI findings.

\footnotetext{
Categories: Radiology, Neurosurgery, Orthopedics

Keywords: lumbar neural foraminal stenosis, degenerative lumbar spinal stenosis, oswestry disability index, back pain
}

\section{Introduction}

Low back pain is highly prevalent and has substantial socioeconomic implications [1]. It is the second most common concern encountered by primary care physicians (after the common cold). Up to $80 \%$ of all individuals will experience low back pain at some point in their lives. Unfortunately, a specific diagnosis is not made in $80 \%$ of low back pain cases [2]. Previous studies have reported a lifetime prevalence of low back pain of $65 \%$ to $80 \%$ [3]. In a local study by Siddiqui et al., the most common cause of low back pain was nerve root compression due to neural foraminal stenosis with a reported prevalence of $73 \%$ [4]. The management of patients with low back pain is either surgical or conservative based on the presence and severity of nerve root compression [5].

Magnetic resonance imaging (MRI) of the lumbar spine is the modality of choice for diagnosing nerve root compression in patients with low back pain with a reported sensitivity and specificity of $80.65 \%$ and $100 \%$, respectively [6]. However, the degree of neural foramen constriction necessary for the symptoms of lumbar spinal stenosis to appear has not been established. Furthermore, the frequency of clinical symptoms (i.e., the patient's disability) and the degree of a radiologically verified constriction have also not been clearly defined [7]. 
Previous studies present conflicting data between anatomic abnormalities of the lumbar spine detected via MRI, clinical history, and patient outcomes. A high prevalence of spine abnormalities was detected via MRI even in asymptomatic patients [1]. These incidental findings might lead to additional testing and carry the potential for unnecessary interventions, increased cost of care, and possibly worse outcomes. One such study shows $15.8 \%$ of total patients showing mild disability, $20.6 \%$ moderate disability, 39.6\% severe disability, $19.04 \%$ crippled, and $5 \%$ of patients bedridden [7]. Therefore, it is important to determine the frequency of severity of disability in patients with neural foraminal stenosis diagnosed via MRI in our clinical settings.

This study aims to determine the frequency of severity of patients' disability as calculated by the Oswestry disability index (ODI) in patients with grade III lumbar neural foraminal stenosis diagnosed on MRI. The study will then ascertain the impact of MRI diagnosis on clinical outcomes compared with careful patient and treatment selection based on clinical scenarios, which may result in reduced diagnoses, unnecessary surgeries, and financial and psychological stress.

\section{Materials And Methods}

This descriptive, cross-sectional study with non-probability, purposive sampling was conducted in the department of radiology for six months. Patients of either gender who were older than 20 years with grade III lumbar neural foraminal stenosis on MRI were included. Patients with a previous history of lumbar spine surgery and those who were getting follow-up scans with already diagnosed nerve root compression were excluded.

Informed consent was obtained. All information was collected through a designed proforma which included the Oswestry disability index (ODI)--a questionnaire containing six statements (denoted levels 0 to 5 ) in each of the 10 sections related to impairments like pain and disabilities such as personal care, lifting, walking, sitting, standing, sleeping, sex life, social life, and traveling. In each section, the patient chose the statement that best described his or her status. ODI is a simple, condition-specific, and preferred multidimensional tool because patients can easily comprehend the form, it is a self-assessment, and it encompasses a wide domain of functions, pain, and limitations as health status [7].

Patients with a score of $0 \%$ to $20 \%$ disability were considered minimally disabled, meaning patients can cope with most living activities. For most minimally disabled patients, no treatment is indicated apart from advice on lifting, sitting, and exercise. A score of $21 \%$ to $40 \%$ meant patients were classified as moderately disabled, and the patient experiences more pain and difficulty with sitting, lifting, and standing. Travel and social life are more difficult, and personal care, sexual activity, and sleeping are not grossly affected. These patients can generally be managed by conservative means. A score of $41 \%$ to $60 \%$ meant patients were considered severely disabled. Severely disabled patients have increased pain intensity that impacts the routines of daily life. These patients require a detailed investigation with the possibility for surgical correction. A score of $61 \%$ to $80 \%$ meant the patient was disabled, and back pain impinged on all aspects of the patient's life, requiring positive intervention. Finally, a score of $81 \%$ to $100 \%$ meant low back pain was so severe that the patient became bedridden.

Date of examination, gender and any previous history of trauma were documented by credentialed MRI technician at the time of examination. Strict exclusion criteria were maintained to control the confounding variables of preexisting trauma or infection.

A Siemens Magnetom 1.5-tesla ( $76 \times 18$ channels; slice thickness, $4.0 \mathrm{~mm}$; gap, $0.4 \mathrm{~mm}$ ) MRI (Siemens AG, Munich, Germany) was used. The MRI radiographic images were interpreted by one experienced radiologist with five years of clinical experience. The patient's disability level was then calculated by calculating the total ODI score, dividing by 50 and multiplying by 100 to calculate the percentage. The frequencies of each level of disability in cases of grade III lumbar neural foraminal stenosis were then calculated accordingly. The statistical analysis was done using IBM SPSS Statistics for Windows, Version 19.0 (IBM Corp, Armonk, $\mathrm{NY}$ ). The frequencies and percentages for categorical variables like age, gender, and severity of patients' disability were calculated. Furthermore, mean and standard deviation for the continuous variables (e.g., age) were also calculated. Stratification was performed with regards to age, gender, and severity of disability to see the effect of the variable on outcomes.

\section{Results}

A total of 250 patients who presented with backache and had MRI examination were included. Of these 250 patients, 27 patients had grade II lumbar neural foraminal stenosis, and 21 had grade I neural foraminal stenosis on MRI and were thus excluded. Thirty-two patients had a spinal infection (e.g., tuberculosis), and 24 patients had a history of trauma. Furthermore, 31 patients were having a follow-up scan for previously diagnosed lumbar neural foraminal stenosis. Hence, after excluding these cases, 115 patients with MRIdiagnosed grade III lumbar neural foraminal stenosis were included in the study (Figure 1). 


\section{Cureus}

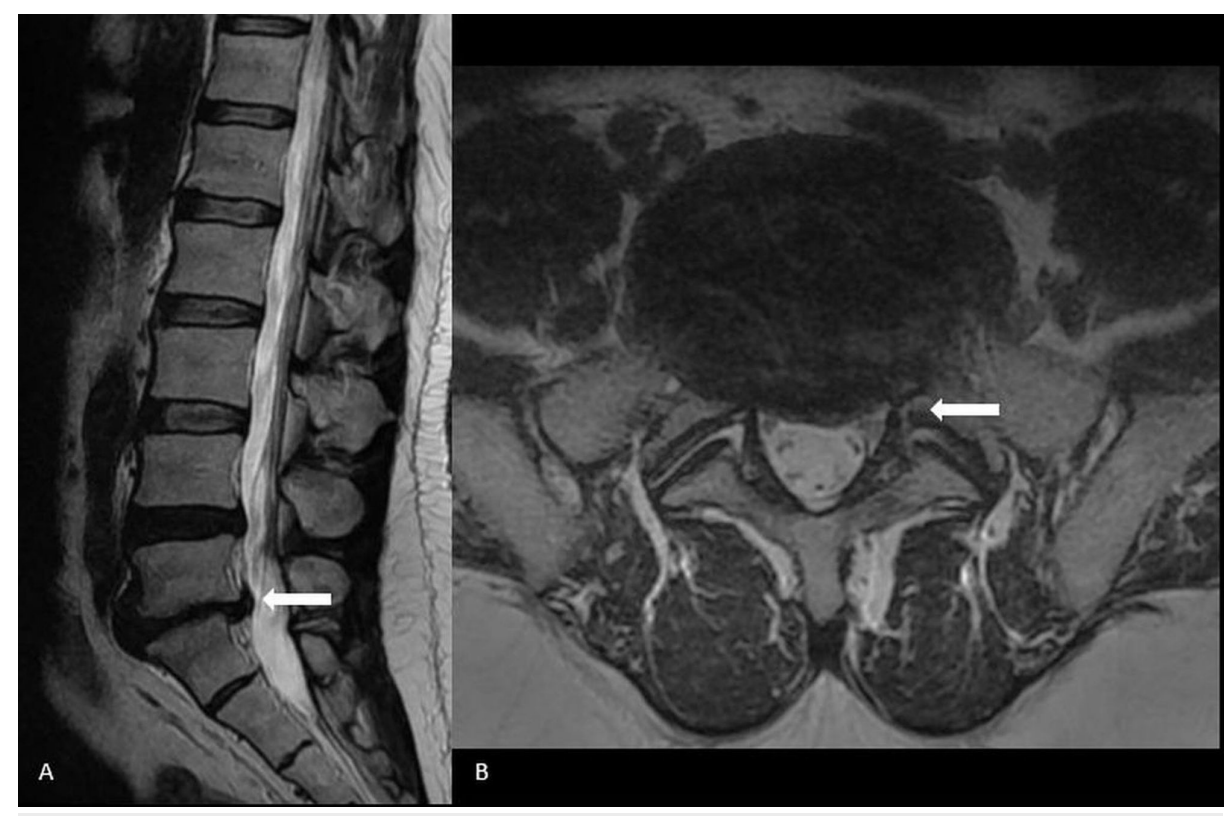

FIGURE 1: L5-S1 disc bulge with moderate thecal sac compression (arrow) on (A) Sagittal and (B) Axial plane.

There were 67 men (58.3\%) and 48 (41.7\%) women. The mean patient age was 51 (range, 20 to 82 years). Most patients ( $\mathrm{n}=34 ; 29.6 \%$ were older than 60 years. Thirty patients (26.1\%) were aged between 50 and 59 years, 31 (27\%) were aged between 40 and 49 years, 11 (9.6\%) patients were aged between 30 and 39 years, and nine $(7.8 \%)$ were aged between 20 and 29 years.

Grade III lumbar neural foraminal stenosis was most common at the L4-L5 level ( $\mathrm{n}=65 ; 56.5 \%)$, followed by the L5-S1 level ( $\mathrm{n}=37 ; 32.2 \%)$, the L3-L4 level ( $\mathrm{n}=9 ; 7.8 \%)$, and the L2-L3 level ( $\mathrm{n}=4 ; 3.5 \%)$.

Most cases of stenosis ( $\mathrm{n}=45 ; 39.1 \%$ ) involved the left side, 40 cases (34.8\%) were bilateral, and 30 (26.1\%) were on the right side. According to the ODI scores, 47 patients (40.9\%) had a severe disability, 32 (27.8\%) had moderate disability, 16 (13.9\%) were disabled, 14 (12.2\%) had a mild disability, and six (5.2\%) were bedridden.

\section{Discussion}

Low back pain is a common health condition affecting approximately $80 \%$ of people at some time in their lives. It is also the most prevalent cause of disability in people under age 45 and has significant socioeconomic implications. Unfortunately, a specific diagnosis cannot be made in approximately $80 \%$ of cases [8]. The most common cause for low back pain is nerve root compression due to neural foraminal stenosis [4].

Management options for low back pain may be mainly either surgical or conservative however long term outcome appear similar in both cases [9]. Surgery intends to decompress the area of narrowing determined on the basis of imaging so as to alleviate pressure on the nerves. This makes accurate analysis important for correct therapy selection [10].

MRI is the imaging modality of choice because of its excellent ability to provide great soft tissue delineation. However, the grade of compression on the thecal sac which is expected to be symptomatic for lumbar spinal stenosis is still not clear. Furthermore, the relationship between the clinical presentation and the imaging findings is still not established. Therefore, it is essential to see the frequency of severity of disability in patients with lumbar neural foraminal stenosis on MRI.

In our study, 69 of 115 patients (60\%) had severe or more than severe disability scores as per the severity of foraminal stenosis seen on MRI. However, the remaining patient population (40\%) showed only mild to moderate disability, far fewer than expected based on radiological assessments. Other studies found similar discrepancies between radiological appearance and clinical symptoms [7,11].

Preoperative evaluation was done by Sirvanci et al. in 63 patients with degenerative spinal stenosis [7]. No definite correlation was seen between radiological stenosis and ODI percentage. Eight patients with severe central stenosis and nine patients with moderate lateral stenosis on imaging demonstrated only minimal 
disability on ODI scores.

Amundsen et al. [11] evaluated 100 patients with backache radiologically and found different types of stenosis; however, no definite relationships could be identified. The imaging findings were much more than expected from the clinical presentation.

Hurri et al. [12] reported a significant correlation between stenosis and ODI percentage, but no such correlation could be recognized in other studies, including those by Jonsson et al. [13], Beattie et al. [14], and Lohman et al. [15]. Moreover, several studies including those by Boden et al. [16] and Szpalski et al. [17] have shown significant lumbar spinal abnormalities on MRI even in completely asymptomatic patients, further proving a lack of correlation between radiologically verified anatomical constriction and clinical picture.

Several theories may explain such discrepancies between the clinical symptoms and radiologically verified anatomical constrictions. One explanation is radiologist overcall; that is, radiologists label morphological changes which may be seen in normal healthy subjects as pathological. Another factor is the impact of positioning on anatomical constriction; extension and flexion movements can result in an increased or decreased cross-sectional diameter of the spinal canal [18]. As in most places, MRI is usually performed with the patient in the supine position; this can result in an underestimation of the spinal canal or foraminal stenosis on static imaging [19].

The most common intervertebral level involved in our patients was L4-L5, which aligned with the reports by other researchers [7, 20-21]. According to many studies the degenerative lumbar spinal stenosis becomes symptomatic usually in the fifth decade of life [22], which also aligns with our findings. We noted a continuous trend of increasing disability with respect to increasing age.

\section{Conclusions}

Although MRI is the imaging modality of choice in cases of lumbar spinal stenosis because of its ability to provide excellent soft tissue resolution, no significant correlation could be demonstrated between imaging appearances and levels of severity of disability in patients with lumbar spinal stenosis in this study and previous studies. In our study, $40 \%$ of patients had only mild to moderate disability implying that their radiological changes were more extensive than expected from the clinical picture. Therefore, degenerative lumbar spinal stenosis is a clinico-radiological syndrome, and treatment decisions should incorporate radiological appearances in addition to the patient's clinical picture before deciding between conservative or surgical management approaches.

\section{Additional Information \\ Disclosures}

Human subjects: Consent was obtained by all participants in this study. Animal subjects: All authors have confirmed that this study did not involve animal subjects or tissue. Conflicts of interest: In compliance with the ICMJE uniform disclosure form, all authors declare the following: Payment/services info: All authors have declared that no financial support was received from any organization for the submitted work. Financial relationships: All authors have declared that they have no financial relationships at present or within the previous three years with any organizations that might have an interest in the submitted work. Other relationships: All authors have declared that there are no other relationships or activities that could appear to have influenced the submitted work.

\section{References}

1. Carrino JA, Lurie JD, Tosteson AN, et al.: Lumbar spine: reliability of MR imaging findings . Radiology. 2009, 250:161-170. 10.1148/radiol.2493071999

2. Brant-Zawadzki MN, Dennis SC, Gade GF, Weinstein MP: Low back pain. Radiology. 2000, 217:321-330. 10.1148/radiology.217.2.r00nv16321

3. Breslau J, Seidenwurm D: Socioeconomic aspects of spinal imaging: impact of radiological diagnosis on lumbar spine-related disability. Magn Reson Imaging. 2000, 11:218-223.

4. Siddiqui AH, Rafique MZ, Ahmad MN, Usman MU: Role of magnetic resonance imaging in lumbar spondylosis. J Coll Physicians Surg Pak. 2005, 15:396-399.

5. Jarvik JG, Deyo RA: Diagnostic evaluation of low back pain with emphasis on imaging . Ann Intern Med. 2002, 1:586-597. 10.7326/0003-4819-137-7-200210010-00010

6. Chawalparit O, Churojana A, Chiewvit P, Thanapipatsir S, Vamvanij V, Charnchaowanish P: The limited protocol MRI in diagnosis of lumbar disc herniation. J Med Assoc Thai. 2006, 89:182-189.

7. Sirvanci M, Bhatia M, Ganiyusufoglu KA, et al.: Degenerative lumbar spinal stenosis: correlation with Oswestry disability index and MR imaging. Eur Spine J. 2008, 17:679-685. 10.1007/s00586-008-0646-5

8. Volinn E: The epidemiology of low back pain in the rest of the world . Spine. 1997, 22:1747-1754.

9. Postacchini F: Results of surgery compared with conservative management for lumbar disc herniations . Spine. 1996, 21:1383-1387.

10. Panagiotis ZE, Athanasios K, Panagiotis D, Minos T, Charis M, Elias L: Functional outcome of surgical treatment for multilevel lumbar spinal stenosis. Acta Orthop. 2006, 77:670-676.

$10.1080 / 17453670610012773$ 
11. Amundsen T, Weber H, Lilleås F, Nordal HJ, Abdelnoor M, Magnaes B: Lumbar spinal stenosis Clinical and radiologic features. Spine. 1995, 15:1178-1186.

12. Hurri H, Slätis P, Soini J, et al.: Lumbar spinal stenosis: assessment of longterm outcome 12 years after operative and conservative treatment. J Spinal Disord. 1998, 11:110-115.

13. Jonsson B, Annertz M, Sjoberg C, Stromqvist B: A prospective and consecutive study of surgically treated lumbar spinal stenosis. Part I: clinical features related to radiographic findings. Spine. 1997, 22:2932-2937.

14. Beattie PF, Meyers SP, Stratford P, Millard RW, Hollenberg GM: Associations between patient report of symptoms and anatomic impairment visible on lumbar magnetic resonance imaging. Spine (Phila Pa 1976). 2000, 1:819-828.

15. Lohman CM, Tallroth K, Kettunen JA, Lindgren KA: Comparison of radiologic signs and clinical symptoms of spinal stenosis. Spine. 2006, 31:1834-1840. 10.1097/01.brs.0000227370.65573.ac

16. Boden SD, Davis DO, Dina TS, Patronas NJ, Wiesel SW: Abnormal magnetic-resonance scans of the lumbar spine in asymptomatic subjects. A prospective investigation. J Bone Joint Surg Am. 1990, 72:403-408.

17. Szpalski M, Gunzburg R: Lumbar spinal stenosis in the elderly: an overview . EurSpine J. 2003, 12:S170-S175. 10.1007/s00586-003-0612-1

18. Penning L, Wilmink JT: Posture-dependent bilateral compression of L4 or L5 nerve roots in facet hypertrophy. A dynamic CT-myelographic study. Spine. 1987, 12:488-500. 10.1097/00007632-19870600000013

19. Weishaupt D, Schmid MR, Zanetti M, et al.: Positional MR imaging of the lumbar spine: does it demonstrate nerve root compromise not visible at conventional MR imaging?. Radiology. 2000, 215:247-253. 10.1148/radiology.215.1.r00ap06247

20. Sigmundsson FG, Kang XP, Jönsson B, Strömqvist B: Correlation between disability and MRI findings in lumbar spinal stenosis A prospective study of 109 patients operated on by decompression. Acta Orthopaedica. 2011, 82:204-210. 10.3109/17453674.2011.566150

21. Kornblum MB, Fischgrund JS, Herkowitz HN, Abraham DA, Berkower DL, Ditkoff JS: Degenerative lumbar spondylolisthesis with spinal stenosis: a prospective long-term study comparing fusion and pseudarthrosis. Spine. 2004, 29:726-733.

22. Atlas SJ, Delitto A: Spinal stenosis: surgical versus nonsurgical treatment. Clin Orthop Relat Res. 2006, 443:198-207. 10.1097/01.blo.0000198722.70138.96 